\title{
Parasitic interference in classical and nulling stellar interferometry
}

 \\ ${ }^{a}$ Max Planck Institut für Radioastronomie, Auf dem Hügel 69, 53121 Bonn, Germany \\ ${ }^{b}$ NASA/GSFC, Greenbelt, MD 20771, USA \\ ${ }^{c}$ Laboratoire Lagrange, CNRS UMR 7293, UNS - Observatoire de la Côte d'Azur BP 4229, \\ F-06304 Nice Cedex 4, France
}

\begin{abstract}
A variety of instrumental effects can corrupt the observable quantities in optical or nulling stellar interferometry. One such effect is parasitic interference, which can occur inside an interferometric instrument. Because of diffraction effects related to beam propagation along finite size optics, or parasitic reflections inside transmitting optics, a coherent crosstalk may occur between the beams and create a parasitic interference pattern superimposed on the genuine one. We developed an analytical approach to describe the impact of this effect on the observables of classical and nulling stellar interferometers. Considering classical interferometry, we show that differential phase and closure phase are both corrupted, depending on the crosstalk level and the residual piston between the beams. Considering typical specifications of piston correction of ground-based interferometers $(\approx 100 \mathrm{~nm})$, the detection of hot Jupiter-like planets by differential phase implies a tolerance on the parasitic flux to about $5 \%$ of the incident intensity. Also, we show that the closure phase relation does not remove this parasitic contribution. The corresponding corrupted closure phase is not zero for an unresolved source, and depends on the residual piston. Considering nulling interferometry, we show that parasitic effects modify the transmission map level, depending on the crosstalk level and the phase shift between primary and secondary beams. In the extreme case of a pi-phase shift, the crosstalk effect implies a decrease of the final output signal-to-noise ratio. Numerical simulations, adapted to handle consistently crosstalk, are then performed to estimate this degradation on a concrete example, the FKSI mission concept.
\end{abstract}

Keywords: parasitic interference, mid-infrared interferometry, phase, closure phase, hot Jupiter, nulling interferometry

\section{INTRODUCTION}

Stellar optical interferometry has substantially evolved in terms of instrument and operation since Fizeau's idea to use this technique for measuring the diameter of $\operatorname{stars}^{1}$. Since Michelson's interferometer, where separated mirrors were fixed on the same telescope mounting, ${ }^{2}$ interferometry with independent telescopes has allowed access to very long baselines ${ }^{3}$ and consequently high angular resolution. In parallel, and considered for the first time by Bracewell ${ }^{4}$ to detect "nonsolar planets", nulling interferometry aims at detecting faint off-axis companions (e.g., planets, exozodiacal disks) orbiting around distant stars. Its principle is to enhance the companion over star flux ratio by producing a destructive interference on the line-of-sight so that the stellar flux is rejected. By an appropriate choice of the baseline length, the flux of the off-axis source can be transmitted and thus detected more easily.

For most of the current classical and nulling interferometers, the beams coming from each telescope, are carried through tunnels up to a combining device. The beams are often reduced in size for practical reasons. However, the transport of these beams through multiple optical modules can be problematic. Because of diffraction effects associated with beam propagation along finite size optics, a coherent cross-talk may occur between beams.

Further author information: (Send correspondence to A.M.) A.M.: $\quad$ E-mail: amatter@mpifr-bonn.mpg.de, D.D.: E-mail: ddefrere@mpifr-bonn.mpg.de, W.D.: $\quad$ E-mail: william.c.danchi@nasa.gov, B.L.: E-mail: lopez@oca.eu, S.L.: E-mail: lagarde@oca.eu, R.P.: E-mail: romain.petrov@unice.fr, M.V.: E-mail: martin.vannier@unice.fr 
This cross-talk implies a parasitic interference between the collection and the recombination steps, so that the "intrinsic" coherence between beams and, consequently, the resulting interferometric observables will be perturbed. Matter et al. ${ }^{5}$ studied for the first time the impact of parasitic interference on the measurement of the complex visibility by a classical two-telescope interferometer. The degradation of the modulus and the phase of the complex visibility depends on two parameters : the residual piston and the contamination rate between interferometric beams. This degradation may be significant when considering the detection by differential phase of close-in extrasolar giant planets. We first extend this study by focussing on the closure phase. This observable has been proposed for the first time by Segransan et al. ${ }^{6}$ to observe challenging targets such as giant extrasolar planets, if more than one interferometric baseline is available. In theory, closure phase entirely removes the atmospheric and instrumental OPD (Optical Path Difference) effects occuring before the recombination of the beams, by summing the pair of baselines over a closed loop, so that the phase shifts between the beam pairs are globally nulled. In our case, we examine the possibility that this observable could be corrupted by parasitic interference and thus could not allow its effects to be removed.

In a second time, we focus on nulling interferometry, knowing that the rejection rate of the stellar flux is usually degraded by various instrumental effects causing an instrumental leakage ${ }^{7}$, in addition to the geometric leakage due to the finite size of the central star. The impact of this instrumental leakage on the detection of faint companions has been considered both experimentally ${ }^{8,9}$, and analytically ${ }^{10}$, but none of these studies addressed the coherent cross-talk that may occur between beams within an interferometric instrument. Considering a Bracewell-like interferometer, we thus study and describe how this instrumental effect perturbs the transmission map of the interferometer, and transforms the geometric and instrumental leakages.

In Section 2, we briefly remind the formalism developed in Matter et al. ${ }^{5}$ and apply it to the closure phase observable. In Section 3, we adapt this formalism to the case of a Bracewell-like interferometer, and derive its perturbed intensity response and the corresponding null output. Then, in Section 4, we quantify the impact of the parasitic interference effect on the hot Jupiter-like sources detection, considering both types of interferometer and their related observables. End-to-end numerical simulations are shown as well. Finally, in Section 5, we summarize our work and discuss how the general problem of crosstalk is handled in the test beds of the nulling interferometer projects, or in the design of the next optical interferometers.

\section{PARASITIC INTERFERENCE IN CLASSICAL INTERFEROMETRY}

\subsection{Interferometric framework}

To create a model of parasitic interference affecting the interferometric observables, especially the phase and the closure phase, we use a three-telescope interferometer characterized by a multi-axial scheme and an image plane recombination. For a general description of the output response of a single baseline interferometer, see also Elias et al. ${ }^{11}$.

First we write the complex amplitudes collected by telescopes 1, 2 and 3, i.e. the three samples of the wavefront, that we respectively note $\psi_{1}, \psi_{2}$ and $\psi_{3}$. We multiply each of them by a real transmission factor noted $\mathrm{t}_{1}, \mathrm{t}_{2}$, and $t_{3}$, which represents the transmission of electric fields through the instrument.

Let us define $\epsilon_{1}$ to be the main fraction of $\psi_{1}$ propagating along the path $1, \epsilon_{12}^{\prime}$ to be the small fraction propagating along the path 2 , and $\epsilon_{13}^{\prime}$ to be the small fraction propagating along the path 3 . We consider the same parasitic effect for $\psi_{2}$, where $\epsilon_{2}$ is the main fraction of $\psi_{2}$ propagating along the path $2, \epsilon_{21}^{\prime}=\epsilon_{12}^{\prime}$ is the small fraction propagating along the path 1 , and $\epsilon_{23}^{\prime}$ is the small fraction propagating along the path 3 . This is the same for $\psi_{3}$, where $\epsilon_{3}$ corresponds to the main fraction of $\psi_{3}$ propagating along the path $3, \epsilon_{31}^{\prime}=\epsilon_{13}^{\prime}$ is the small fraction propagating along the path 1 , and $\epsilon_{32}^{\prime}=\epsilon_{23}^{\prime}$ is the small fraction propagating along the path 2. Fig. 1 shows two examples of cross-talk between beams. A possible imbalance of parasitic flux between paths, not presented in this paper, was considered in the study but appeared to be a second order parameter. All the cross-talk occuring inside the instrument produces a resulting parasitized pattern in a conjugate of the pupil plane, located just before the recombination on the detector. This is described in the following equation giving the complex amplitude preceding the recombination of beams 1 and 2 :

$$
\psi_{\text {pup }, 12}(x, y)=\left[\epsilon_{1} t_{1} \psi_{1}+\epsilon_{12}^{\prime} t_{2} \psi_{2}+\epsilon_{13}^{\prime} t_{3} \psi_{3}\right] \mathrm{P}\left(x-\frac{b_{12}}{2}, y\right)+\left[\epsilon_{2} t_{2} \psi_{2}+\epsilon_{12}^{\prime} t_{1} \psi_{1}+\epsilon_{23}^{\prime} t_{3} \psi_{3}\right] \mathrm{P}\left(x+\frac{b_{12}}{2}, y\right) .
$$

Here $x$ and $y$ are the coordinates in the pupil plane. $b_{12}$ is the distance between the pupils of telescopes 1 and 2 , which are reduced in size at the entrance of the interferometer, $D$ being the diameter of these pupils. $\mathrm{P}(x, y)=$ 


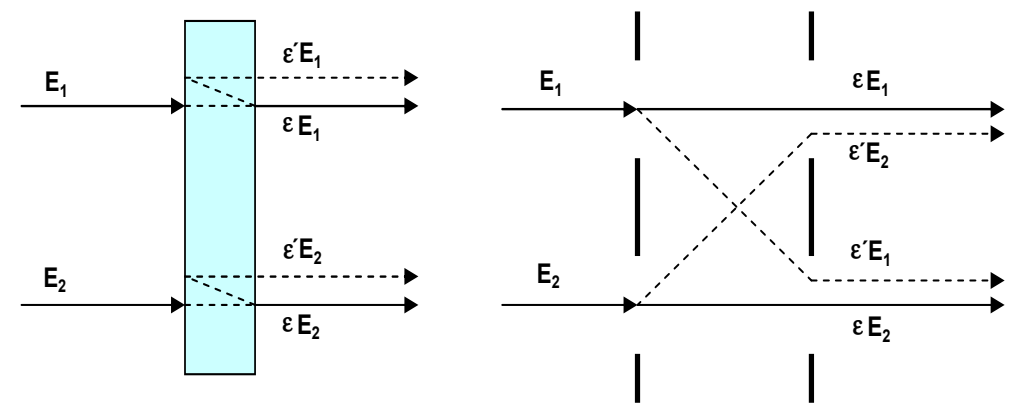

Figure 1. Simple scheme describing the different possibilities of beam contamination (or crosstalk). This beam contamination can be produced by a parasitic reflection inside transmitting optics (left figure) or by a beam mixing (right figure). $\epsilon E_{1}$ ans $\epsilon E_{2}$ represent the main beams (in electrical field), while $\epsilon^{\prime} E_{1}$ ans $\epsilon^{\prime} E_{2}$ corresponds to the secondary beams reflected inside the optics and/or going though the wrong paths (for example due to diffraction or beam mixing).

$\Pi\left(\frac{\sqrt{x^{2}+y^{2}}}{D}\right)$, where $\Pi(x, y)$ is the transmission function of a circular aperture with a uniform transmission of 1 inside and 0 outside. Considering similarly $b_{13}$ and $b_{23}$ the distance between the pupils of telescopes 1 and 2 , and 2 and 3, respectively, the complex amplitude preceding the recombination of beam 1 with beam 3 , and beam 2 with beam 3 is given by:

$$
\begin{aligned}
& \psi_{\text {pup }, 13}(x, y)=\left[\epsilon_{1} t_{1} \psi_{1}+\epsilon_{12}^{\prime} t_{2} \psi_{2}+\epsilon_{13}^{\prime} t_{3} \psi_{3}\right] \mathrm{P}\left(x-\frac{b_{13}}{2}, y\right)+\left[\epsilon_{3} t_{3} \psi_{3}+\epsilon_{23}^{\prime} t_{2} \psi_{2}+\epsilon_{13}^{\prime} t_{1} \psi_{1}\right] \mathrm{P}\left(x+\frac{b_{13}}{2}, y\right), \\
& \psi_{\text {pup }, 23}(x, y)=\left[\epsilon_{2} t_{2} \psi_{2}+\epsilon_{12}^{\prime} t_{1} \psi_{1}+\epsilon_{23}^{\prime} t_{3} \psi_{3}\right] \mathrm{P}\left(x-\frac{b_{23}}{2}, y\right)+\left[\epsilon_{3} t_{3} \psi_{3}+\epsilon_{13}^{\prime} t_{1} \psi_{1}+\epsilon_{23}^{\prime} t_{2} \psi_{2}\right] \mathrm{P}\left(x+\frac{b_{23}}{2}, y\right) .
\end{aligned}
$$

Here we assumed that the three pupils are aligned along the x-axis of the pupil plane. From Eqs. 1, 2 and 3, we discuss how parasitic fringes are formed on the detector.

As we will see later, the complex degree of coherence between the different parasitized beams will become:

$$
\begin{aligned}
& <\left[\epsilon_{1} t_{1} \psi_{1}+\epsilon_{12}^{\prime} t_{2} \psi_{2}+\epsilon_{13}^{\prime} t_{3} \psi_{3}\right]\left[\epsilon_{2} t_{2} \psi_{2}+\epsilon_{12}^{\prime} t_{1} \psi_{1}+\epsilon_{23}^{\prime} t_{3} \psi_{3}\right]^{*}>, \\
& <\left[\epsilon_{1} t_{1} \psi_{1}+\epsilon_{12}^{\prime} t_{2} \psi_{2}+\epsilon_{13}^{\prime} t_{3} \psi_{3}\right]\left[\epsilon_{3} t_{3} \psi_{3}+\epsilon_{23}^{\prime} t_{2} \psi_{2}+\epsilon_{13}^{\prime} t_{1} \psi_{1}\right]^{*}>, \\
& <\left[\epsilon_{2} t_{2} \psi_{2}+\epsilon_{12}^{\prime} t_{1} \psi_{1}+\epsilon_{23}^{\prime} t_{3} \psi_{3}\right]\left[\epsilon_{3} t_{3} \psi_{3}+\epsilon_{13}^{\prime} t_{1} \psi_{1}+\epsilon_{23}^{\prime} t_{2} \psi_{2}\right]^{*}>,
\end{aligned}
$$

instead of the corresponding 'intrinsic' coherence terms $\left.<\psi_{1} \psi_{2}^{*}\right\rangle,\left\langle\psi_{1} \psi_{3}^{*}\right\rangle$, and $\left\langle\psi_{2} \psi_{3}^{*}\right\rangle$.

\subsection{Interference and formation of parasitic fringes}

In order to describe the formation of parasitic fringes, we consider the most general case of the observation of an extended source. The vector $\boldsymbol{\alpha}$ is the angular coordinate in the plane of the sky. The telescopes are located at $\gamma_{1}, \gamma_{\mathbf{2}}$ and $\gamma_{\mathbf{3}}, \gamma$ being the coordinate in the plane containing the telescopes and counted in units of wavelength $\left(\gamma=\frac{\mathbf{r}}{\lambda}\right.$ ) (see Fig. 1). $\frac{\boldsymbol{B}_{12}}{\lambda}=\gamma_{1}-\gamma_{2}, \frac{\boldsymbol{B}_{13}}{\lambda}=\gamma_{1}-\gamma_{\mathbf{3}}, \frac{\boldsymbol{B}_{23}}{\lambda}=\gamma_{2}-\gamma_{\mathbf{3}}$, with $\boldsymbol{B}_{12}, \boldsymbol{B}_{13}$, and $\boldsymbol{B}_{23}$ the corresponding baselines, respectively. The electric field emitted by each point of the extended source, located at $\boldsymbol{\alpha}$, is represented by its amplitude $\mathrm{A}(\alpha)$ and its phase $\phi(\boldsymbol{\alpha}, \mathrm{t})$ :

$$
\psi_{\mathrm{S}}(\boldsymbol{\alpha})=\mathrm{A}(\boldsymbol{\alpha}) e^{i[\omega t+\phi(\boldsymbol{\alpha}, t)]} \mathrm{d} \boldsymbol{\alpha} .
$$

In the plane of the telescopes, the phase shift of the wavefront emitted by each point of the source and measured on the $\gamma$ position is $\Delta \Phi=-2 \pi \boldsymbol{\alpha} \cdot \gamma$. Therefore, when considering the contributions of all the emitting points of the source, each telescope observes a packet of wavefronts (respectively $\psi_{1}, \psi_{2}$, and $\psi_{3}$ ) :

$$
\begin{aligned}
& \psi_{1}=\int \mathrm{A}(\boldsymbol{\alpha}) e^{i[\omega t+\phi(\boldsymbol{\alpha}, t)]} e^{i 2 \pi \boldsymbol{\alpha} \cdot \boldsymbol{\gamma}_{1}} d \boldsymbol{\alpha}, \\
& \psi_{2}=\int \mathrm{A}(\boldsymbol{\alpha}) e^{i[\omega t+\phi(\boldsymbol{\alpha}, t)]} e^{i 2 \pi \boldsymbol{\alpha} \cdot \boldsymbol{\gamma}_{\mathbf{2}}} d \boldsymbol{\alpha}, \\
& \psi_{3}=\int \mathrm{A}(\boldsymbol{\alpha}) e^{i[\omega t+\phi(\boldsymbol{\alpha}, t)]} e^{i 2 \pi \boldsymbol{\alpha} \cdot \boldsymbol{\gamma}_{3}} d \boldsymbol{\alpha} .
\end{aligned}
$$


We can now write the expression of the complex amplitudes in the detector plane by performing the Fourier transform of Eqs. 1, 2 and 3 with respect to $x$ and $y$. In the detector plane, where $\beta$ and $\eta$ are the conjugate angular coordinates related to $x$ and $y$, and $\hat{\mathrm{P}}(\beta, \eta)$ is the pupil diffraction function, we obtain :

$$
\begin{aligned}
& W_{12}(\beta, \eta)=\left(\epsilon_{1} t_{1} \psi_{1}+\epsilon_{12}^{\prime} t_{2} \psi_{2}+\epsilon_{13}^{\prime} t_{3} \psi_{3}\right) \hat{\mathrm{P}}(\beta, \eta) e^{\frac{-i \pi b \beta}{\lambda}}+\left(\epsilon_{2} t_{2} \psi_{2}+\epsilon_{12}^{\prime} t_{1} \psi_{1}+\epsilon_{23}^{\prime} t_{3} \psi_{3}\right) \hat{\mathrm{P}}(\beta, \eta) e^{\frac{i \pi b \beta}{\lambda}}=X_{1}+X_{2} . \\
& W_{13}(\beta, \eta)=\left(\epsilon_{1} t_{1} \psi_{1}+\epsilon_{12}^{\prime} t_{2} \psi_{2}+\epsilon_{13}^{\prime} t_{3} \psi_{3}\right) \hat{\mathrm{P}}(\beta, \eta) e^{\frac{-i \pi b \beta}{\lambda}}+\left(\epsilon_{3} t_{3} \psi_{3}+\epsilon_{23}^{\prime} t_{2} \psi_{2}+\epsilon_{13}^{\prime} t_{1} \psi_{1}\right) \hat{\mathrm{P}}(\beta, \eta) e^{\frac{i \pi b \beta}{\lambda}}=X_{1}+X_{3} \\
& W_{23}(\beta, \eta)=\left(\epsilon_{2} t_{2} \psi_{2}+\epsilon_{12}^{\prime} t_{1} \psi_{1}+\epsilon_{23}^{\prime} t_{3} \psi_{3}\right) \hat{\mathrm{P}}(\beta, \eta) e^{\frac{-i \pi b \beta}{\lambda}}+\left(\epsilon_{3} t_{3} \psi_{3}+\epsilon_{13}^{\prime} t_{1} \psi_{1}+\epsilon_{23}^{\prime} t_{2} \psi_{2}\right) \hat{\mathrm{P}}(\beta, \eta) e^{\frac{i \pi b \beta}{\lambda}}=X_{2}+X_{3} .
\end{aligned}
$$

The terms containing $\psi_{1}, \psi_{2}$ and $\psi_{3}$ are not affected by the Fourier transform since $\psi_{1}, \psi_{2}$, and $\psi_{3}$ can be considered, in a good approximation, as constant over the aperture telescope area. This assumption is equivalent to the requirement that the source is not resolved by the telescopes themselves or that the field of view is small. On the detector, we observe the three following intensity patterns :

$$
\begin{aligned}
& I_{12}(\beta, \eta)=<\left|X_{1}\right|^{2}>+<\left|X_{2}\right|^{2}>+2 \operatorname{Re}<X_{1} X_{2}^{*}>, \\
& I_{13}(\beta, \eta)=<\left|X_{1}\right|^{2}>+<\left|X_{3}\right|^{2}>+2 \operatorname{Re}<X_{1} X_{3}^{*}>, \\
& I_{23}(\beta, \eta)=<\left|X_{2}\right|^{2}>+<\left|X_{3}\right|^{2}>+2 \operatorname{Re}<X_{2} X_{3}^{*}>.
\end{aligned}
$$

The photometric terms, $<\left|X_{1}\right|^{2}>,<\left|X_{2}\right|^{2}>$, and $<\left|X_{3}\right|^{2}>$ are equal to:

$$
\begin{aligned}
<\left|X_{1}\right|^{2}> & =\hat{\mathrm{P}}^{2}(\beta, \eta)\left[\left(\epsilon_{1}^{2} t_{1}^{2}+\epsilon_{12}^{\prime 2} t_{2}^{2}+\epsilon_{13}^{\prime 2} t_{3}^{2}\right) \hat{\mathrm{O}}(0)\right]+2 \epsilon_{1} \epsilon_{12}^{\prime} t_{1} t_{2} \operatorname{Re}\left(\hat{\mathrm{O}}\left(\gamma_{\mathbf{1}}-\gamma_{\mathbf{2}}\right)\right) \\
& +2 \epsilon_{1} \epsilon_{13}^{\prime} t_{1} t_{3} \operatorname{Re}\left(\hat{\mathrm{O}}\left(\gamma_{\mathbf{1}}-\gamma_{\mathbf{3}}\right)\right)+2 \epsilon_{12}^{\prime} \epsilon_{23}^{\prime} t_{2} t_{3} \operatorname{Re}\left(\hat{\mathrm{O}}\left(\gamma_{\mathbf{2}}-\gamma_{\mathbf{3}}\right)\right), \\
<\left|X_{2}\right|^{2}> & =\hat{\mathrm{P}}^{2}(\beta, \eta)\left[\left(\epsilon_{2}^{2} t_{2}^{2}+\epsilon_{12}^{\prime 2} t_{1}^{2}+\epsilon_{23}^{\prime 2} t_{3}^{2}\right) \hat{\mathrm{O}}(0)\right]+2 \epsilon_{2} \epsilon_{12}^{\prime} t_{1} t_{2} \operatorname{Re}\left(\hat{\mathrm{O}}\left(\gamma_{\mathbf{2}}-\gamma_{\mathbf{1}}\right)\right) \\
& +2 \epsilon_{2} \epsilon_{23}^{\prime} t_{2} t_{3} \operatorname{Re}\left(\hat{\mathrm{O}}\left(\gamma_{\mathbf{2}}-\gamma_{\mathbf{3}}\right)\right)+2 \epsilon_{12}^{\prime} \epsilon_{23}^{\prime} t_{1} t_{3} \operatorname{Re}\left(\hat{\mathrm{O}}\left(\gamma_{\mathbf{1}}-\gamma_{\mathbf{3}}\right)\right), \\
<\left|X_{3}\right|^{2}> & =\hat{\mathrm{P}}^{2}(\beta, \eta)\left[\left(\epsilon_{3}^{2} t_{3}^{2}+\epsilon_{13}^{\prime 2} t_{1}^{2}+\epsilon_{23}^{\prime 2} t_{2}^{2}\right) \hat{\mathrm{O}}(0)\right]+2 \epsilon_{3} \epsilon_{13}^{\prime} t_{1} t_{3} \operatorname{Re}\left(\hat{\mathrm{O}}\left(\gamma_{\mathbf{3}}-\gamma_{\mathbf{1}}\right)\right) \\
& +2 \epsilon_{3} \epsilon_{23}^{\prime} t_{2} t_{3} \operatorname{Re}\left(\hat{\mathrm{O}}\left(\gamma_{\mathbf{2}}-\gamma_{\mathbf{3}}\right)\right)+2 \epsilon_{13}^{\prime} \epsilon_{23}^{\prime} t_{1} t_{2} \operatorname{Re}\left(\hat{\mathrm{O}}\left(\gamma_{\mathbf{1}}-\gamma_{\mathbf{2}}\right)\right) .
\end{aligned}
$$

Here $\mathrm{O}(\boldsymbol{\alpha})=\mathrm{A}^{2}(\boldsymbol{\alpha})$ is the intensity distribution of the source and $\hat{\mathrm{O}}\left(\boldsymbol{\gamma}_{\mathbf{1}}-\boldsymbol{\gamma}_{\mathbf{2}}\right)=<\psi_{1} \psi_{2}^{*}>=\int \mathrm{O}(\boldsymbol{\alpha}) e^{-i 2 \pi \boldsymbol{\alpha} \cdot\left(\boldsymbol{\gamma}_{\mathbf{1}}-\boldsymbol{\gamma}_{\mathbf{2}}\right)} \mathrm{d} \boldsymbol{\alpha}$ is its Fourier transform at the spatial frequency $\left(\gamma_{\mathbf{1}}-\gamma_{\mathbf{2}}\right)$, also called the complex degree of mutual coherence, is : $\hat{\mathrm{O}}\left(\boldsymbol{\gamma}_{\mathbf{1}}-\boldsymbol{\gamma}_{\mathbf{2}}\right)=<\psi_{1} \psi_{2}^{*}>=\int \mathrm{O}(\boldsymbol{\alpha}) e^{-i 2 \pi \boldsymbol{\alpha} \cdot\left(\boldsymbol{\gamma}_{\mathbf{1}}-\boldsymbol{\gamma}_{\mathbf{2}}\right)} \mathrm{d} \boldsymbol{\alpha} . \mathrm{O}(\boldsymbol{\alpha})$ is a real function and its Fourier transform can also be noted $\hat{\mathrm{O}}\left(\gamma_{\mathbf{1}}-\gamma_{\mathbf{2}}\right)=\rho_{12} \mathrm{e}^{i \phi_{12}}$ (or $\hat{\mathrm{O}}\left(\gamma_{\mathbf{1}}-\gamma_{\mathbf{3}}\right)=\rho_{13} \mathrm{e}^{i \phi_{13}}$, or $\left.\hat{\mathrm{O}}\left(\boldsymbol{\gamma}_{\mathbf{2}}-\boldsymbol{\gamma}_{\mathbf{3}}\right)=\rho_{23} \mathrm{e}^{i \phi_{23}}\right)$. However the intrinsic phase of the source measured by the different baselines is always perturbed by an additive term representing the atmospheric perturbations and instrumental instabilities occuring before recombination of the beams. Therefore the phase observable produced by each pair of beams can be written again as:

$$
\begin{aligned}
& \Phi_{12}=\phi_{12}+\phi_{12, o p d}, \\
& \Phi_{13}=\phi_{13}+\phi_{13, \text { opd }}, \\
& \Phi_{23}=\phi_{23}+\phi_{23, \text { opd }},
\end{aligned}
$$

with, for example, $\phi_{12, \text { opd }}=\frac{2 \pi}{\lambda} \delta_{12}=\frac{2 \pi}{\lambda}\left(\delta_{1}-\delta_{2}\right)$ where $\delta_{12}$ is the OPD between beams 1 and 2 . Then, in the following, we will consider the phase terms $\Phi_{12}, \Phi_{13}$, and $\Phi_{23}$, containing the astrophysical contribution and the instrumental and atmospheric effects occuring before beam recombination.

\subsection{Parasitic differential and closure phases}

From the general correlation terms $\left\langle\mathrm{X}_{1} \mathrm{X}_{2}^{*}\right\rangle,\left\langle\mathrm{X}_{1} \mathrm{X}_{3}^{*}\right\rangle$, and $\left\langle\mathrm{X}_{2} \mathrm{X}_{3}^{*}\right\rangle$, we extract the resulting parasitized phase terms $\chi_{12}, \chi_{13}$, and $\chi_{23}$ : 


$$
\begin{aligned}
& <\mathrm{X}_{1} \mathrm{X}_{2}^{*}>=\hat{\mathrm{P}}^{2}(\beta, \eta)\left[\left(\epsilon_{1} \epsilon_{12}^{\prime} t_{1}^{2}+\epsilon_{2} \epsilon_{12}^{\prime} t_{2}^{2}+\epsilon_{12}^{\prime} \epsilon_{23}^{\prime} t_{3}^{2}\right) \hat{\mathrm{O}}(0)+\epsilon_{1} \epsilon_{2} t_{1} t_{2} \hat{\mathrm{O}}\left(\gamma_{1}-\gamma_{\mathbf{2}}\right)+\epsilon_{1} \epsilon_{23}^{\prime} t_{1} t_{3} \hat{\mathrm{O}}^{*}\left(\gamma_{1}-\gamma_{\mathbf{3}}\right)\right. \\
& +\epsilon_{12}^{\prime 2} t_{1} t_{2} \hat{\mathrm{O}}^{*}\left(\gamma_{2}-\gamma_{1}\right)+\epsilon_{12}^{\prime} \epsilon_{23}^{\prime} t_{2} t_{3} \hat{\mathrm{O}}^{*}\left(\gamma_{2}-\gamma_{3}\right) \\
& \left.+\epsilon_{13}^{\prime} \epsilon_{2} t_{2} t_{3} \hat{O}^{*}\left(\gamma_{\mathbf{3}}-\gamma_{\mathbf{2}}\right)+\epsilon_{13}^{\prime} \epsilon_{12}^{\prime} t_{3} t_{1} \hat{\mathrm{O}}^{*}\left(\gamma_{\mathbf{3}}-\gamma_{\mathbf{1}}\right)\right] e^{-2 i \pi b \frac{\beta}{\lambda}} \\
& =\hat{\mathrm{P}}^{2}(\beta, \eta)\left[\rho_{12} e^{i \chi_{12}}\right] e^{-2 i \pi b \frac{\beta}{\lambda}}=\hat{\mathrm{P}}^{2}(\beta, \eta) \rho_{12} e^{-i\left(2 \pi b \frac{\beta}{\lambda}-\chi_{12}\right)}, \\
& <\mathrm{X}_{1} \mathrm{X}_{3}^{*}>=\hat{\mathrm{P}}^{2}(\beta, \eta)\left[\left(\epsilon_{1} \epsilon_{13}^{\prime} t_{1}^{2}+\epsilon_{3} \epsilon_{13}^{\prime} t_{3}^{2}+\epsilon_{12}^{\prime} \epsilon_{23}^{\prime} t_{2}^{2}\right) \hat{\mathrm{O}}(0)+\epsilon_{1} \epsilon_{3} t_{1} t_{3} \hat{\mathrm{O}}\left(\gamma_{1}-\gamma_{3}\right)+\epsilon_{1} \epsilon_{23}^{\prime} t_{1} t_{2} \hat{\mathrm{O}}^{*}\left(\gamma_{1}-\gamma_{2}\right)\right. \\
& +\epsilon_{13}^{\prime 2} t_{3} t_{1} \hat{\mathrm{O}}^{*}\left(\gamma_{\mathbf{3}}-\gamma_{1}\right)+\epsilon_{12}^{\prime} \epsilon_{3} t_{2} t_{3} \hat{\mathrm{O}}^{*}\left(\gamma_{\mathbf{2}}-\gamma_{\mathbf{3}}\right) \\
& \left.+\epsilon_{12}^{\prime} \epsilon_{13}^{\prime} t_{2} t_{1} \hat{\mathrm{O}}^{*}\left(\gamma_{2}-\gamma_{1}\right)+\epsilon_{12}^{\prime} \epsilon_{23}^{\prime} t_{3} t_{2} \hat{\mathrm{O}}^{*}\left(\gamma_{2}-\gamma_{3}\right)\right] e^{-2 i \pi b \frac{\beta}{\lambda}} \\
& =\hat{\mathrm{P}}^{2}(\beta, \eta)\left[\rho_{13} e^{i \chi_{13}}\right] e^{-2 i \pi b \frac{\beta}{\lambda}}=\hat{\mathrm{P}}^{2}(\beta, \eta) \rho_{13} e^{-i\left(2 \pi b \frac{\beta}{\lambda}-\chi_{13}\right)}, \\
& <\mathrm{X}_{2} \mathrm{X}_{3}^{*}>=\hat{\mathrm{P}}^{2}(\beta, \eta)\left[\left(\epsilon_{2} \epsilon_{23}^{\prime} t_{2}^{2}+\epsilon_{3} \epsilon_{23}^{\prime} t_{3}^{2}+\epsilon_{12}^{\prime} \epsilon_{13}^{\prime} t_{1}^{2}\right) \hat{\mathrm{O}}(0)+\epsilon_{2} \epsilon_{3} t_{2}^{2} \hat{\mathrm{O}}\left(\gamma_{\mathbf{2}}-\gamma_{\mathbf{3}}\right)+\epsilon_{2} \epsilon_{13}^{\prime} t_{1} t_{2} \hat{\mathrm{O}}^{*}\left(\gamma_{2}-\gamma_{\mathbf{1}}\right)\right. \\
& +\epsilon_{23}^{\prime 2} t_{3} t_{2} \hat{\mathrm{O}}^{*}\left(\gamma_{3}-\gamma_{2}\right)+\epsilon_{12}^{\prime} \epsilon_{3} t_{1} t_{3} \hat{\mathrm{O}}^{*}\left(\gamma_{\mathbf{1}}-\gamma_{\mathbf{3}}\right) \\
& \left.+\epsilon_{12}^{\prime} \epsilon_{23}^{\prime} t_{2} t_{1} \hat{\mathrm{O}}^{*}\left(\gamma_{\mathbf{1}}-\gamma_{\mathbf{2}}\right)+\epsilon_{23}^{\prime} \epsilon_{13}^{\prime} t_{3} t_{1} \hat{\mathrm{O}}^{*}\left(\gamma_{\mathbf{3}}-\gamma_{\mathbf{1}}\right)\right] e^{-2 i \pi b \frac{\beta}{\lambda}} \\
& =\hat{\mathrm{P}}^{2}(\beta, \eta)\left[\rho_{23} e^{i \chi_{23}}\right] e^{-2 i \pi b \frac{\beta}{\lambda}}=\hat{\mathrm{P}}^{2}(\beta, \eta) \rho_{23} e^{-i\left(2 \pi b \frac{\beta}{\lambda}-\chi_{23}\right)} \text {. }
\end{aligned}
$$

The resulting expression of the parasitized phase terms is the following:

$$
\begin{aligned}
& \chi_{12}=\operatorname{atan}\left[\frac{\alpha_{12} \sin \left(\Phi_{12}\right)+\alpha_{13} \sin \left(\Phi_{13}\right)+\alpha_{23} \sin \left(\Phi_{23}\right)}{\beta_{12} \cos \left(\Phi_{12}\right)+\beta_{13} \cos \left(\Phi_{13}\right)+\beta_{23} \cos \left(\Phi_{23}\right)+\beta \hat{O}(0)}\right], \\
& \chi_{13}=\operatorname{atan}\left[\frac{\gamma_{13} \sin \left(\Phi_{13}\right)+\gamma_{12} \sin \left(\Phi_{12}\right)+\gamma_{23} \sin \left(\Phi_{23}\right)}{\delta_{13} \cos \left(\Phi_{13}\right)+\delta_{12} \cos \left(\Phi_{12}\right)+\delta_{23} \cos \left(\Phi_{23}\right)+\delta \hat{O}(0)}\right], \\
& \chi_{23}=\operatorname{atan}\left[\frac{\eta_{23} \sin \left(\Phi_{23}\right)+\eta_{12} \sin \left(\Phi_{12}\right)+\eta_{13} \sin \left(\Phi_{13}\right)}{\kappa_{23} \cos \left(\Phi_{23}\right)+\kappa_{12} \cos \left(\Phi_{12}\right)+\kappa_{13} \cos \left(\Phi_{13}\right)+\kappa \hat{O}(0)}\right],
\end{aligned}
$$

with

$$
\begin{aligned}
& \alpha_{12}=\left(1-\epsilon_{12}^{\prime \prime 2}\right) t_{12} \rho_{12}, \alpha_{13}=\left(\epsilon_{23}^{\prime \prime}-\epsilon_{13}^{\prime \prime} \epsilon_{12}^{\prime \prime}\right) t_{12} t_{32} \rho_{13}, \alpha_{23}=\left(\epsilon_{12}^{\prime \prime} \epsilon_{23}^{\prime \prime}-\epsilon_{13}^{\prime \prime}\right) t_{32} \rho_{23} . \\
& \beta_{12}=\left(1+\epsilon_{12}^{\prime \prime 2}\right) t_{12} \rho_{12}, \beta_{13}=\left(\epsilon_{23}^{\prime \prime}+\epsilon_{13}^{\prime \prime} \epsilon_{12}^{\prime \prime}\right) t_{12} t_{32} \rho_{13}, \beta_{23}=\left(\epsilon_{12}^{\prime \prime} \epsilon_{23}^{\prime \prime}+\epsilon_{13}^{\prime \prime}\right) t_{32} \rho_{23}, \beta=\left(\epsilon_{12}^{\prime \prime}\left(1+t_{12}^{2}\right)+\epsilon_{13}^{\prime \prime} \epsilon_{23}^{\prime \prime} t_{32}^{2}\right) \\
& \gamma_{13}=\left(1-\epsilon_{13}^{\prime \prime}\right) t_{12} t_{32} \rho_{13}, \gamma_{12}=\left(\epsilon_{23}^{\prime \prime}-\epsilon_{13}^{\prime \prime} \epsilon_{12}^{\prime \prime}\right) t_{12} \rho_{12}, \gamma_{23}=\left(\epsilon_{12}^{\prime \prime}-\epsilon_{13}^{\prime \prime} \epsilon_{23}^{\prime \prime}\right) t_{32} \rho_{23} . \\
& \delta_{13}=\left(1+\epsilon_{13}^{\prime \prime 2}\right) t_{12} t_{32} \rho_{13}, \delta_{12}=\left(\epsilon_{23}^{\prime \prime}+\epsilon_{13}^{\prime \prime} \epsilon_{12}^{\prime \prime}\right) t_{12} \rho_{12}, \delta_{23}=\left(\epsilon_{12}^{\prime \prime}+\epsilon_{13}^{\prime \prime} \epsilon_{23}^{\prime \prime}\right) t_{32} \rho_{23}, \delta=\left(\epsilon_{13}^{\prime \prime} t_{12}^{2}+\epsilon_{12}^{\prime \prime} \epsilon_{23}^{\prime \prime}+\epsilon_{13}^{\prime \prime} t_{32}^{2}\right) \\
& \eta_{23}=\left(1-\epsilon_{23}^{\prime \prime 2}\right) t_{32} \rho_{23}, \eta_{12}=\left(\epsilon_{23}^{\prime \prime} \epsilon_{12}^{\prime \prime}-\epsilon_{13}^{\prime \prime}\right) t_{12} \rho_{12}, \eta_{13}=\left(\epsilon_{12}^{\prime \prime}-\epsilon_{13}^{\prime \prime} \epsilon_{23}^{\prime \prime}\right) t_{12} t_{32} \rho_{13} . \\
& \kappa_{23}=\left(1+\epsilon_{23}^{\prime \prime 2}\right) t_{32} \rho_{23}, \kappa_{12}=\left(\epsilon_{13}^{\prime \prime}+\epsilon_{12}^{\prime \prime} \epsilon_{23}^{\prime \prime}\right) t_{12} \rho_{12}, \kappa_{13}=\left(\epsilon_{12}^{\prime \prime}+\epsilon_{13}^{\prime \prime} \epsilon_{23}^{\prime \prime}\right) t_{32} t_{12} \rho_{13}, \kappa=\left(\epsilon_{23}^{\prime \prime}+\epsilon_{12}^{\prime \prime} \epsilon_{13}^{\prime \prime} t_{12}^{2}+\epsilon_{23}^{\prime \prime} t_{32}^{2}\right) .
\end{aligned}
$$

Here $t_{12}=\frac{t_{1}}{t_{2}}$ and $t_{32}=\frac{t_{3}}{t_{2}}$ are the transmission ratio between beam paths. $\epsilon^{\prime \prime}=\frac{\epsilon^{\prime}}{\epsilon}$ represents the percentage of parasitic contribution (or contamination rate), evaluated with respect to $\epsilon$ which is the fraction of the electric field propagating along the right path. $\epsilon^{\prime \prime 2}=\left(\frac{\epsilon^{\prime}}{\epsilon}\right)^{2}$ is the equivalent ratio in terms of flux (or intensity), called 'parasitic flux factor' in the following. Since the $\chi$ terms are defined as parasitized phases, the parasitic phases, representing the parasitic contributions added to the phase terms $\Phi_{12}, \Phi_{13}$, and $\Phi_{23}$, are $\chi_{12}-\Phi_{12}, \chi_{13}-\Phi_{13}$, and $\chi_{23}-\Phi_{23}$, respectively.

In our three-telescope scheme, we assumed a closed loop configuration for the baselines, so that we can define the closure phase observable $\psi$ by :

$$
\psi=\Phi_{12}+\Phi_{23}-\Phi_{13}
$$

This allows to remove the atmospheric and instrumental effects occuring before recombination since, in this closed loop configuration, $\phi_{12, \text { opd }}+\phi_{23, \text { opd }}-\phi_{13, \text { opd }}=0$. Assuming that the post-recombination instrumental effects are removed by devices such as the BCD (see e.g. Vannier ${ }^{12}$ ), then, we would finally have :

$$
\psi=\phi_{12}+\phi_{23}-\phi_{13}
$$


In presence of parasitic fringes, the phase closure relation applies to the parasitized phase terms $\chi_{12}, \chi_{13}$, and $\chi_{23}$. The parasitized closure phase, denoted as $X$, is thus given by:

$$
X=\chi_{12}+\chi_{23}-\chi_{13}
$$

Let us explicit this expression to see if the phase closure relation can still allow us to remove the atmospheric and instrumental contributions contained in $\Phi_{12}, \Phi_{13}$, and $\Phi_{23}$, along with the parasitic contribution affecting them. The parasitized closure phase becomes:

$$
X=\operatorname{atan}\left(X_{12}\right)+\operatorname{atan}\left(X_{23}\right)-\operatorname{atan}\left(X_{13}\right)
$$

with

$$
\begin{aligned}
& X_{12}=\frac{\alpha_{12} \sin \left(\Phi_{12}\right)+\alpha_{13} \sin \left(\Phi_{13}\right)+\alpha_{23} \sin \left(\Phi_{23}\right)}{\beta_{12} \cos \left(\Phi_{12}\right)+\beta_{13} \cos \left(\Phi_{13}\right)+\beta_{23} \cos \left(\Phi_{23}\right)+\beta \hat{O}(0)} \\
& X_{23}=\frac{\eta_{23} \sin \left(\Phi_{23}\right)+\eta_{12} \sin \left(\Phi_{12}\right)+\eta_{13} \sin \left(\Phi_{13}\right)}{\kappa_{23} \cos \left(\Phi_{23}\right)+\kappa_{12} \cos \left(\Phi_{12}\right)+\kappa_{13} \cos \left(\Phi_{13}\right)+\kappa \hat{O}(0)} \\
& X_{13}=\frac{\gamma_{13} \sin \left(\Phi_{13}\right)+\gamma_{12} \sin \left(\Phi_{12}\right)+\gamma_{23} \sin \left(\Phi_{23}\right)}{\delta_{13} \cos \left(\Phi_{13}\right)+\delta_{12} \cos \left(\Phi_{12}\right)+\delta_{23} \cos \left(\Phi_{23}\right)+\delta \hat{O}(0)}
\end{aligned}
$$

Knowing that $\operatorname{atan}(x)+\operatorname{atan}(y)=\operatorname{atan}\left(\frac{x+y}{1-x y}\right)$, we can develop the previous expression of the parasitized closure phase $X$ to obtain:

$$
X=\operatorname{atan}\left[\frac{X_{12}+X_{23}-X_{13}+X_{12} X_{23} X_{13}}{1-X_{12} X_{23}+X_{12} X_{13}+X_{23} X_{13}}\right]
$$

Regarding the dependencies of $X_{12}, X_{23}$, and $X_{13}$ on the contamination rates $\left(\epsilon_{12}^{\prime \prime}, \epsilon_{13}^{\prime \prime}\right.$, and $\left.\epsilon_{23}^{\prime \prime}\right)$ and the cosine and sine of the phase terms $\left(\Phi_{12}, \Phi_{13}\right.$, and $\left.\Phi_{23}\right)$, it clearly appears that we cannot retrieve the intrinsic closure phase relation of the astrophysical source, defined by: $\psi=\phi_{12}+\phi_{23}-\phi_{13}$. Especially, the parasitized closure phase of an unresolved source is not zero and depends on both the different contamination rates and the residual piston through the $\phi_{\text {opd }}$ terms contained in the phase terms $\Phi_{12}, \Phi_{23}$, and $\Phi_{13}$.

\subsection{Amplitude of parasitic differential phase}

In this section, we remind some of the results described in Matter et al. ${ }^{5}$ concerning the estimation of the impact of parasitic interference in the context of the observation of different "synthetic" stellar systems with a hot Jupiter, in $\mathrm{L}$ band. Table 1 shows the parameters of the different hot Jupiter synthetic spectra used here. In this context let us consider a stellar system with a planet. We respectively note $I_{*}(\lambda)$ and $I_{\text {pl }}(\lambda)$ the

Table 1. Values of various parameters discriminating each phase spectrum extracted from Barman et al. ${ }^{13}$.

\begin{tabular}{lccc}
\hline & Type of star & $T_{\text {int }}$ & Separation star/planet \\
\hline spectrum 1 & $\mathrm{dM} 5$ & $500 \mathrm{~K}$ & $0.05 \mathrm{AU}$ \\
spectrum 2 & $\mathrm{dM} 5$ & $500 \mathrm{~K}$ & $0.5 \mathrm{AU}$ \\
spectrum 3 & $\mathrm{dM} 5$ & $1000 \mathrm{~K}$ & $0.005 \mathrm{AU}$ \\
spectrum 4 & $\mathrm{dM} 5$ & $1000 \mathrm{~K}$ & $0.1 \mathrm{AU}$ \\
spectrum 5 & $\mathrm{G} 2$ & $500 \mathrm{~K}$ & $0.3 \mathrm{AU}$ \\
spectrum 6 & $\mathrm{G} 2$ & $500 \mathrm{~K}$ & $1 \mathrm{AU}$ \\
spectrum 7 & $\mathrm{G} 2$ & $1000 \mathrm{~K}$ & $0.05 \mathrm{AU}$ \\
spectrum 8 & $\mathrm{G} 2$ & $1000 \mathrm{~K}$ & $1 \mathrm{AU}$ \\
\hline
\end{tabular}

monochromatic flux of the two components separated by an angular distance $\boldsymbol{\alpha}_{\mathbf{p l}}$. The star appears as a disc 
of constant angular diameter $\mathrm{D}_{*}$, and the planet appears as a point-like source. The parasitized phase of such a system, noted $\chi_{\text {syst }}(\lambda)$, writes as :

$$
\chi_{\mathrm{syst}}(\lambda)=\arctan \frac{t_{12}\left(1-\epsilon^{\prime \prime 2}\right)\left[C_{*} I_{*} \sin \left(\Phi_{*}\right)+I_{\mathrm{pl}} \sin \left(\Phi_{*}+\Phi_{\mathrm{pl}}\right)\right]}{\epsilon^{\prime \prime}\left(1+t_{12}^{2}\right)\left(I_{*}+I_{\mathrm{pl}}\right)+t_{12}\left(1+\epsilon^{\prime \prime 2}\right)\left[C_{*} I_{*} \cos \left(\Phi_{*}\right)+I_{\mathrm{pl}} \cos \left(\Phi_{*}+\Phi_{\mathrm{pl}}\right)\right]} .
$$

, with $\Phi_{\mathrm{pl}}(\lambda)=2 \pi \boldsymbol{u} \cdot \boldsymbol{\alpha}_{\mathrm{pl}}$ and $\Phi_{*}(\lambda)=\frac{2 \pi}{\lambda} \delta_{*}$, where $\delta_{*}$ represents the residual piston uncorrected by the fringe tracking device. The intrinsic visibility of the star is $C_{*}(\mathrm{u})$. The u-axis is chosen along the interferometric baseline defined above, and takes the value $\mathbf{u}=\frac{\mathbf{B}}{\lambda}$. We consider the amplitude in $\mathrm{L}$ band of the phase signature from the planet, $a_{\text {syst }}=\max \left(\Phi_{\text {syst }}(\lambda)\right)-\min \left(\Phi_{\text {syst }}(\lambda)\right)$, and of the corresponding parasitic phase, $a_{\text {par }}=$ $\max \left(\chi_{\text {syst }}(\lambda)-\Phi_{\text {syst }}(\lambda)\right)-\min \left(\chi_{\text {syst }}(\lambda)-\Phi_{\text {syst }}(\lambda)\right)$. These quantities are calculated for a large range of $\delta_{*}$ values and are represented with respect to the parasitic flux factor $\epsilon^{\prime \prime 2}$ in Fig.2. The $\lambda / 500$ and $\lambda / 30$ cases give a very similar parasitic phase amplitude that can only be distinguished for the spectra 5,6 and 7 . It appears that if typical fringe specifications are observed, the planet signal (horizontal solid line) always lies above the parasitic phase amplitude; a hot Jupiter detection, with an intrinsic phase amplitude exceeding by a factor of three the parasitic one, is thus achieved with a parasitic intensity equal to $5 \%$ of the total intensity.

\section{PARASITIC INTERFERENCE IN NULLING INTERFEROMETRY}

\subsection{Parasitic interference model}

To create a model of parasitic interference, we consider the case of a two-telescope pupil plane Bracewell interferometer. Its intensity response (or transmission map) is derived from the addition of the complex amplitude of the electric fields, coming from a point-like source located at the $\vec{\theta}$ direction in the sky (see Fig.3), collected by each telescope and splitted to go either in the destructive or the constructive output, namely $E_{1}(\vec{\theta})$ and $E_{2}(\vec{\theta})$. However, we here assume a flux contamination (crosstalk), resulting from secondary reflections inside the tramsitting optics or mutual contaminations between those two beams, as already described in Matter et al. $^{5}$. This flux contamination results in parasitic interferences. We define $\epsilon$ as the main fraction of $E_{1}(\vec{\theta})$ (resp. $\left.E_{2}(\vec{\theta})\right)$ propagating along the path 1 (resp. 2). Then, we define $\epsilon^{\prime}$ as the small fraction of $E_{1}(\vec{\theta})$, either reflected inside the transmitting optics and still following the path 1 , or having contaminated $E_{2}(\vec{\theta})$ by crosstalk and following the path 2 (see Fig.1). We assume the same parasitic effect for $E_{2}(\vec{\theta})$. All the parasitic reflections and/or cross talk occuring inside the instrument produces a resulting perturbed pattern in the overlapping pupil plane. At any point $\vec{r}$ of this plane, the total electric field is thus given by:

$$
E(\vec{\theta}, \vec{r})=\Pi\left(\frac{r}{D}\right)\left[\epsilon E_{1}(\vec{\theta}) e^{i \phi_{1}}+\epsilon^{\prime} E_{2}(\vec{\theta}) e^{i \phi_{2}^{\prime}}+\epsilon E_{2}(\vec{\theta}) e^{i \phi_{2}}+\epsilon^{\prime} E_{1}(\vec{\theta}) e^{i \phi_{1}^{\prime}}\right]
$$

where $\Pi\left(\frac{r}{D}\right)$ is the transmission function of the pupil, $r=\sqrt{x^{2}+y^{2}}$ is the distance in the pupil plane, $D$ is the diameter of the pupil, $\phi_{k}$ and $\phi_{k}^{\prime}$ are the corresponding phase terms of the main part and the secondary part of the beam $k$, respectively.

\subsection{Perturbed intensity response}

From Eq. 33, we derive in the following the perturbed intensity response of the nulling interferometer, noted $\tilde{R}_{\lambda}(\vec{\theta})$, in the presence of cross talk. The expression of the complex electric field $E_{k}(\vec{\theta})$ is $E_{k}(\vec{\theta})=E_{k} e^{i \frac{2 \pi}{\lambda}} \vec{x}_{k} \cdot \vec{\theta}$, where $\vec{x}_{k}$ is the position of the telescope $k$. We thus have:

$$
\begin{aligned}
E(\vec{\theta}, \vec{r}) & =\Pi\left(\frac{r}{D}\right)\left[\epsilon\left(E_{1} e^{i\left(\frac{2 \pi}{\lambda} \vec{x}_{1} \cdot \vec{\theta}+\phi_{1}\right)}+E_{2} e^{\left(i \frac{2 \pi}{\lambda} \vec{x}_{2} \cdot \vec{\theta}+\phi_{2}\right)}\right)+\epsilon^{\prime}\left(E_{1} e^{\left(i \frac{2 \pi}{\lambda} \vec{x}_{1} \cdot \vec{\theta}+\phi_{1}^{\prime}\right)}+E_{2} e^{\left(i \frac{2 \pi}{\lambda} \vec{x}_{2} \cdot \vec{\theta}+\phi_{2}^{\prime}\right)}\right)\right], \\
& =\Pi\left(\frac{r}{D}\right) \tilde{r}_{\lambda}(\vec{\theta}) .
\end{aligned}
$$



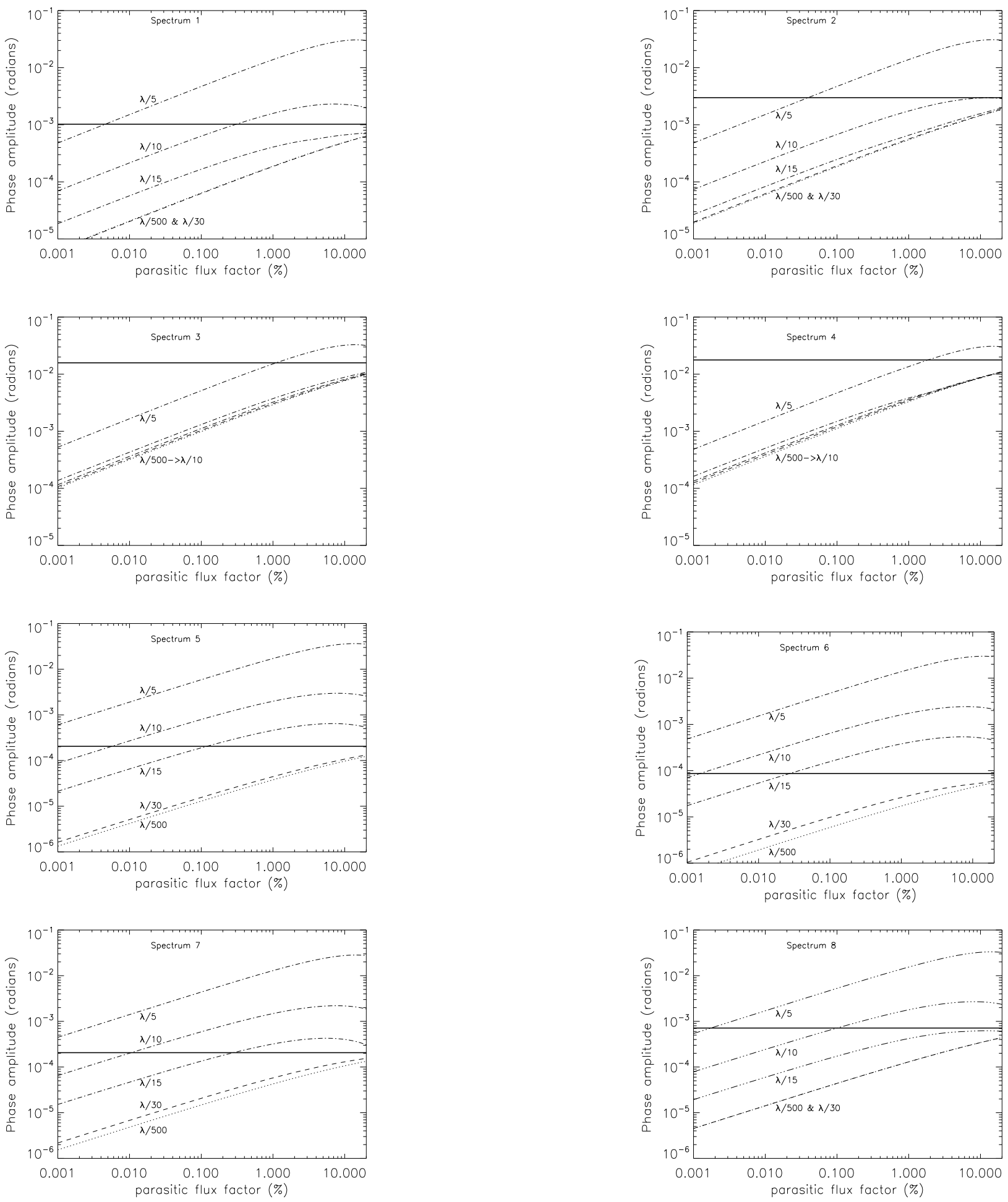

Figure 2. Amplitude, in L band, of the intrinsic phase of a stellar system with a hot Jupiter, $a_{\text {syst }}$ (horizontal solid line), and amplitude in L band of the related parasitic phase, $a_{\text {par }}$ (other dashed and dotted lines), plotted with respect to $\epsilon^{\prime \prime 2}$. Different values of $\delta_{*}$ ranging from $\lambda / 500$ to $\lambda / 5$ are considered, and each panel is related to a different hot Jupiter spectrum. The $\lambda / 500$ and $\lambda / 30$ cases correspond approximately to the typical specifications that would be respectively achieved by a spatial and a ground-based fringe tracking device $\left(\delta_{*} \approx 2 \mathrm{~nm}\right.$ and $\left.100 \mathrm{~nm}\right)$. 


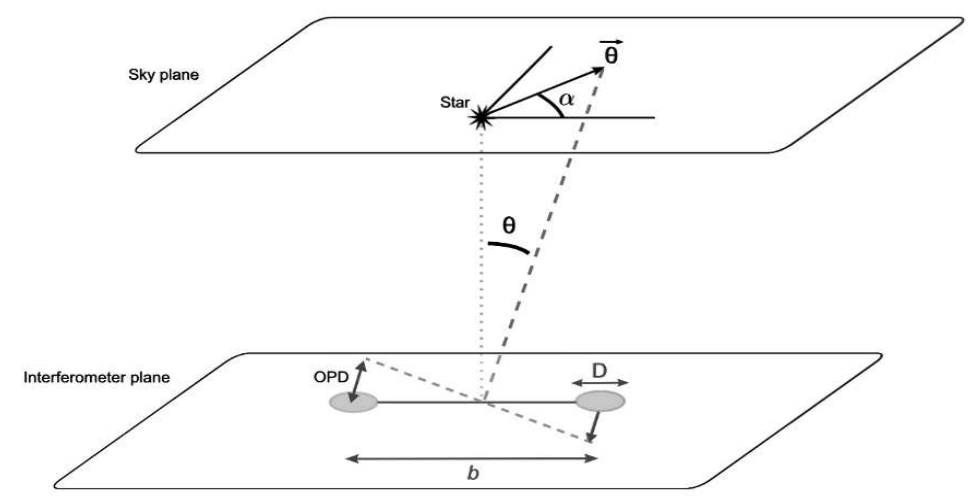

Figure 3. Geometric configuration of the interferometer and the astrophysical source. The two angular coordinates $(\theta, \alpha)$ give the position in the sky plane. Here, the line-of-sight is assumed to be perpendicular to the interferometer plane.

Then we calculate the perturbed intensity response of the interferometer:

$$
\begin{aligned}
\tilde{R}_{\lambda}(\vec{\theta}) & =\tilde{r}_{\lambda}(\vec{\theta}) \tilde{r}_{\lambda}^{*}(\vec{\theta}) \\
& =\epsilon^{2}\left[\left|E_{1}\right|^{2}+\left|E_{2}\right|^{2}+2 E_{1} E_{2} \cos \left(\left(\phi_{1}-\phi_{2}\right)+\frac{2 \pi}{\lambda}\left(\overrightarrow{x_{1}}-\overrightarrow{x_{2}}\right) \cdot \vec{\theta}\right)\right] \\
& +\epsilon \epsilon^{\prime}\left[2\left|E_{1}\right|^{2} \cos \left(\phi_{1}-\phi_{1}^{\prime}\right)+2\left|E_{2}\right|^{2} \cos \left(\phi_{2}-\phi_{2}^{\prime}\right)+2 E_{1} E_{2}\left(\cos \left(\frac{2 \pi}{\lambda}\left(\overrightarrow{x_{1}}-\overrightarrow{x_{2}}\right) \cdot \vec{\theta}+\left(\phi_{1}^{\prime}-\phi_{2}\right)\right)\right.\right. \\
& \left.\left.+\cos \left(\frac{2 \pi}{\lambda}\left(\overrightarrow{x_{1}}-\overrightarrow{x_{2}}\right) \cdot \vec{\theta}+\left(\phi_{1}-\phi_{2}^{\prime}\right)\right)\right)\right] \\
& +\epsilon^{\prime 2}\left[\left|E_{1}\right|^{2}+\left|E_{2}\right|^{2}+2 E_{1} E_{2} \cos \left(\left(\phi_{1}^{\prime}-\phi_{2}^{\prime}\right)+\frac{2 \pi}{\lambda}\left(\overrightarrow{x_{1}}-\overrightarrow{x_{2}}\right) \cdot \vec{\theta}\right)\right]
\end{aligned}
$$

Assuming that each beam is divided into two equal parts by the balanced beam splitter, and that they were collected by two telescopes of unitary size, we have $\left|E_{1}\right|^{2}=\left|E_{2}\right|^{2}=1 / 2$. Following the notations of Fig. 3, we have $\left(\vec{x}_{1}-\vec{x}_{2}\right) \cdot \vec{\theta}=b \theta \cos (\alpha)$. The general expression of the perturbed intensity response then becomes:

$$
\begin{aligned}
\tilde{R}_{\lambda}(\theta, \alpha) & =\epsilon^{2}\left[1+\cos \left(\left(\phi_{1}-\phi_{2}\right)+\pi \frac{b \theta}{\lambda} \cos (\alpha)\right)\right] \\
& +\epsilon \epsilon^{\prime}\left[\cos \left(\phi_{1}-\phi_{1}^{\prime}\right)+\cos \left(\phi_{2}-\phi_{2}^{\prime}\right)+\cos \left(\pi \frac{b \theta}{\lambda} \cos (\alpha)+\left(\phi_{1}^{\prime}-\phi_{2}\right)\right)\right. \\
& \left.+\cos \left(\pi \frac{b \theta}{\lambda} \cos (\alpha)+\left(\phi_{1}-\phi_{2}^{\prime}\right)\right)\right] \\
& +\epsilon^{\prime 2}\left[1+\cos \left(\left(\phi_{1}^{\prime}-\phi_{2}^{\prime}\right)+\pi \frac{b \theta}{\lambda} \cos (\alpha)\right)\right]
\end{aligned}
$$

We clearly see from the expression above that two parasitic contributions are degrading the intrinsic intensity response of the interferometer, which is $\epsilon^{2}\left[1+\cos \left(\left(\phi_{1}-\phi_{2}\right)+\frac{2 \pi}{\lambda}\left(\overrightarrow{x_{1}}-\overrightarrow{x_{2}}\right) \cdot \vec{\theta}\right)\right]$. From this general expression $\tilde{R}_{\lambda}$, the phase shift terms between the different beam parts can be simplified if we assume a symmetric design for the instrument, thus implying symmetric contaminations. In that case, we can simplify the phase shift terms :

$$
\begin{aligned}
& \phi_{1}-\phi_{2}=\phi_{1}^{\prime}-\phi_{2}^{\prime} \\
& \phi_{1}-\phi_{1}^{\prime}=\phi_{2}-\phi_{2}^{\prime}
\end{aligned}
$$

From these simplifications, we assume that, after beam mixing or parasitic reflection, any secondary beam has been identically phase-shifted of $\Delta \phi$ with respect to the primary beams before recombination. More precisely, we will consider $\phi_{k}-\phi_{k}^{\prime}=\Delta \phi$. In the following, we explicit the expression of $\tilde{R}_{\lambda}$ with respect to the value of 
the main phase shift between the primary beams $\phi_{1}-\phi_{2}$.

Following the basic principle of nulling interferometry, a relative $\pi$-phase shift is applied between the two beams before recombination, namely $\phi_{1}-\phi_{2}=\pi$ radians. In addition, instrumental phase perturbation could indeed occur for instance because of vibrations inside the interferometer arms or imperfect piston correction by the fringe tracker. Therefore, a variable instrumental phase shift $\delta \phi(t)$ is also affecting the beams in the interferometric arms. Therefore, at the destructive output of the beam splitter, the phase shifts between the different parts of the beams are now:

$$
\begin{aligned}
& \phi_{1}-\phi_{2}=\phi_{1}^{\prime}-\phi_{2}^{\prime}=\pi+\delta \phi(t) \\
& \phi_{1}-\phi_{1}^{\prime}=\phi_{2}-\phi_{2}^{\prime}=\Delta \phi
\end{aligned}
$$

The perturbed intensity response thus writes as:

$$
\tilde{R}_{\lambda}(\theta, \alpha)=\left[2\left(\epsilon^{2}+\epsilon^{\prime 2}\right)+4 \epsilon \epsilon^{\prime} \cos (\Delta \phi)\right] \sin ^{2}\left(\pi \frac{b \theta}{\lambda} \cos (\alpha)+\frac{\delta \phi}{2}\right)
$$

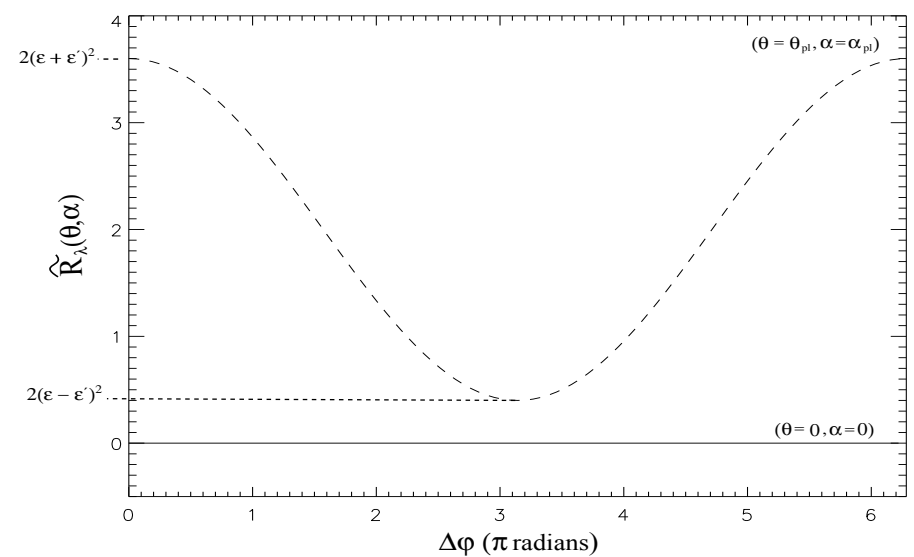

Figure 4. Evolution of the perturbed transmission map, $\tilde{R}_{\lambda}(\theta, \alpha)$, at $10 \mu \mathrm{m}$, as a function of the phase shift $\Delta \phi$ between the primary and secondary beams. No instrumental phase shift is considered here, and two angular positions are shown: on-axis for the stellar source (solid line), and off-axis $\left(\theta_{\mathrm{pl}}, \alpha_{\mathrm{pl}}\right)$ for an hypothetical planet (dashed line), assuming $\sin ^{2}\left(\pi \frac{b \theta_{\mathrm{pl}}}{\lambda} \cos \left(\alpha_{\mathrm{pl}}\right)\right) \approx 1$ for the planet.

From this expression, we clearly see that the effect of the beam contamination on the transmission map depends on the value of $\Delta \phi$ (see Fig.4). In the unlikely situation where no phase shift is produced by the contamination process, namely $\Delta \phi=0$, the transmission map becomes : $R(\lambda, \vec{\theta})=2\left(\epsilon+\epsilon^{\prime}\right)^{2} \sin ^{2}\left(\pi \frac{b \theta}{\lambda} \cos (\alpha)+\frac{\delta \phi}{2}\right)$. In this case, the primary and secondary parts of each beam meet in a co-phased way in the overlapping pupil plane. This is represented by the factor $\left(\epsilon+\epsilon^{\prime}\right)^{2}$. In contrast, considering $\Delta \phi=\pi$, the perturbed intensity response becomes:

$$
\tilde{R}_{\lambda}(\theta, \alpha)=2\left(\epsilon-\epsilon^{\prime}\right)^{2} \sin ^{2}\left(\pi \frac{b \theta}{\lambda} \cos (\alpha)+\frac{\delta \phi}{2}\right)
$$

In this case, we can clearly notice a decrease of the overall level of the transmission map of the interferometer by a factor $\left(\epsilon-\epsilon^{\prime}\right)^{2}$, at the destructive output. The flux coming from the on-axis stellar source is thus better rejected, while the flux of the off-axis source is less transmitted. This result is not surprising if we refer to the parasitic interference phenomenon extensively described in Matter et al. ${ }^{5}$. For instance, in the case of crosstalk occuring in a classical interferometer, parasitic interference creates two other fringe patterns in addition to the intrinsic fringe pattern due to the astrophysical source. One of them can be assimilated to a Young-like fringe pattern, which is created by two sources perfectly coherent represented by the main part of the beam (noted $\epsilon$ ) and the small contribution having contaminated the other path (noted $\epsilon^{\prime}$ ). This fringe pattern does not contain any information about the astrophysical visibility and phase of the source, and artificially increases the proportion of 
coherent flux in the resulting interference pattern, since a part of the incoherent flux of the source was actually used to form these Young-like fringes. In our case, the apparent improvement of the on-axis source nulling is thus accompanied by a lower transmission of the flux of the off-axis source. The signal coded in the perturbed interferogram and forming the transmission map, does not refer anymore to the frequential information of the whole astrophysical source at $B / \lambda$, but also to a contribution from the lower spatial frequencies resulting from the transfert function of individual apertures. To summarize, a part of the low-frequency energy is mixed with the high-frequency contributions at $B / \lambda$. This mixing generates a confusion in the frequential signature of the astrophysical object.

\subsection{Perturbed null output}

The intensity response projected on the plane of the sky forms the transmission map of the interferometer. We here assume that the final detection is done in an image plane. Therefore, an image similar to that of a single telescope is formed, except that the contribution of each source is affected by the intensity response of the interferometer depending on its location. No fringe is formed nor recorded, and the final output generally consists of the total intensity in the diffraction limited field of view, namely the size of the Airy pattern. Following the mathematical description of $\mathrm{Absil}^{14}$, this final output or "null" then writes :

$$
F(\lambda)=T(\lambda) \iint\left[B_{\mathrm{sky}}(\lambda, \theta, \alpha) \tilde{R}_{\lambda}(\theta, \alpha)+B_{\mathrm{bckg}}(\lambda, \theta, \alpha, t)\right] P(\theta, \alpha) \theta d \theta d \alpha,
$$

with $T(\lambda)$ the wavelength-dependent total transmission of the interferometer, $B_{\text {sky }}(\lambda, \theta, \alpha)$ the brightness distribution of the source in the diffraction-limited field of view, $B_{\mathrm{bckg}}(\lambda, \theta, \alpha, t)$ the time-dependent brightness of the incoherent background emission (sky thermal emission, telescope, optical train), $P(\theta, \alpha)$ the point spread function of a single telescope, and finally $\tilde{R}_{\lambda}(\theta, \alpha)$ the transmission map of the interferometer affected by parasitic interference. Here, we consider a magnification factor of 1 , so that we keep the same angular coordinates in the focal plane, namely $(\alpha, \theta)$.

Let us consider the observation of an extended source, composed of a star partially resolved by the interferometer and a secondary component (planet, exozodiacal disk, ...). The corresponding brightness distribution on the sky is:

$$
B_{\mathrm{sky}}(\lambda, \theta, \alpha)=B_{*}(\lambda) \Pi\left(\frac{\sqrt{\theta^{2}+\alpha^{2}}}{\theta_{*}}\right)+B_{\mathrm{co}}(\lambda) f(\theta, \alpha) .
$$

$\Pi\left(\frac{\sqrt{\theta^{2}+\alpha^{2}}}{\theta_{*}}\right)$ is the top-hat function, which is equal to 1 in the angular domain $\left[0, \theta_{*}\right]$, where $\theta_{*}$ is the angular diameter of the stellar photosphere, and equal to 0 outside. The stellar brightness per square meter per steradian $B_{*}(\lambda)$ is considered to be constant over the stellar surface. $B_{\mathrm{co}}(\lambda)$ is the brightness of the secondary component, while $f(\theta, \alpha)$ is its distribution on the sky.

Assuming that the stellar angular diameter is small compared to the fringe spacing $\left(\theta_{*} \ll \frac{\lambda}{b}\right)$, and that $\delta \phi(t) \ll 1$, we can simplify the expression of the perturbed transmission map in the angular domain $\left[0, \theta_{*}\right]$ as:

$$
\tilde{R}_{\theta_{*}}(\lambda, \theta, \alpha) \approx\left(2\left(\epsilon^{2}+\epsilon^{\prime 2}\right)+4 \epsilon \epsilon^{\prime} \cos (\Delta \phi)\right)\left(\pi \frac{b \theta}{\lambda} \cos (\alpha)+\frac{\delta \phi(t)}{2}\right)^{2}
$$

Then, considering that the finite stellar photosphere of angular radius $\theta_{*}$ is almost unresolved by one single telescope, as well as the secondary component of the system, we can assume $P(\theta, \alpha)=\frac{2 J_{1}\left(\pi \sqrt{\theta^{2}+\alpha^{2}} D / \lambda\right)}{\left.\pi \sqrt{\theta^{2}+\alpha^{2}} D / \lambda\right)} \approx 1$ for both sources. The final output can then be written as:

$$
\begin{aligned}
F(\lambda) & \approx B_{*}(\lambda) \int_{0}^{2 \pi} \int_{0}^{\theta_{*}}\left[\left(2\left(\epsilon^{2}+\epsilon^{\prime 2}\right)+4 \epsilon \epsilon^{\prime} \cos (\Delta \phi)\right)\left(\pi \frac{b \theta}{\lambda} \cos (\alpha)+\frac{\delta \phi(t)}{2}\right)^{2}\right] \theta d \theta d \alpha \\
& +B_{\mathrm{co}}(\lambda) \iint \tilde{R}(\lambda, \theta, \alpha) f(\theta, \alpha) \theta d \theta d \alpha+\iint B_{\mathrm{bckg}}(\lambda, \theta, \alpha, t) \theta d \theta d \alpha .
\end{aligned}
$$

This perturbed null output contains, in addition to the secondary component and background signals, the stellar leakage. This term can be written as :

$$
F_{*}(\lambda) \approx B_{*}(\lambda)\left(\left(\epsilon^{2}+\epsilon^{\prime 2}\right)+2 \epsilon \epsilon^{\prime} \cos (\Delta \phi)\right)\left[\frac{\pi^{3} b^{2} \theta_{*}^{4}}{2 \lambda^{2}}+\frac{\delta \phi^{2}(t)}{2} \pi \theta_{*}^{2}\right]
$$


where the first term represents the classical geometric stellar leakage, and the second term can be assimilated to the instrumental leakage, which is related to the instrumental variable phase shift $\delta \phi(t)$. Both terms are multiplied by the parasitic factor $\left(\epsilon^{2}+\epsilon^{\prime 2}\right)+2 \epsilon \epsilon^{\prime} \cos (\Delta \phi)$, which modifies the leakage level.

\subsection{Noise and bias analysis}

In this section, we detail the impact of cross talk on the sensitivity of a nulling interferometer, using the expression of the perturbed geometric and instrumental leakage expressions derived above. The contribution of the backgroung emission in the null output is not taken into account in this section since it is independent from the level of cross talk (see Eq. 41). To estimate the maximum degradation caused by the crosstalk effect, we place in the case where $\Delta \phi=\pi$, so that we have: $\left(\epsilon^{2}+\epsilon^{\prime 2}\right)+2 \epsilon \epsilon^{\prime} \cos (\Delta \phi)=\left(\epsilon-\epsilon^{\prime}\right)^{2}$. After detection of the signal by a rotating interferometer, the geometric leakage is removed since it is not modulated by the rotation of the interferometer. The remaining bias is then reduced to the part of the instrumental leakage, which is not removed by the rotation (see Lay ${ }^{10}$ for more details). To estimate the detection efficiency of a nulling interferometer, we thus define a 'signal to noise and bias' ratio, hereafter noted $S N B$, taking into account the stellar leakage photon noise and the instrumental bias. This estimator is the ratio between the useful 'signal', i.e. the flux of the secondary component that should be measured at the destructive output during an integration time $\Delta t$, and the sum of the instrumental bias and the photon noise, also measured during the same integration time $\Delta t$ :

$$
\begin{aligned}
\text { Signal } & =B_{\text {co }}(\lambda) \Delta t \iint \tilde{R}_{\lambda}(\theta, \alpha) f(\theta, \alpha) \theta d \theta d \alpha, \\
\text { Noise } & =\sqrt{B_{*}(\lambda) \Delta t\left(\epsilon-\epsilon^{\prime}\right)^{2}\left(\frac{\sigma_{\delta \phi}^{2}}{2} \pi \theta_{*}^{2}+\frac{\pi^{3} b^{2} \theta_{*}^{4}}{2 \lambda^{2}}\right),} \\
\text { Bias } & =B_{*}(\lambda) \Delta t\left(\frac{\sigma_{\delta \phi}^{2}}{2} \pi \theta_{*}^{2}\right)
\end{aligned}
$$

$\sigma_{\delta \phi}$ is the standard deviation of the instrumental phase shift error, $\delta \phi(t)$, affecting the beams during the integration time $\Delta t$. Thus our estimator writes as:

$$
S N B=\frac{\text { Signal }}{\text { Noise }+ \text { Bias }}
$$

In order to simplify the quantitative study of the problem, we will consider in the following that the total incident energy is conserved during beam contamination, so that $\epsilon^{2}+\epsilon^{\prime 2}=1$. The amplitude of the parasitic effect will only depends on the flux contamination rate, namely the cross talk level $\epsilon^{\prime 2}$.

\subsection{Quantitative estimation}

We here calculate the amplitude of the degradation of the 'signal to noise and bias' ratio, $S N B$, as a function of $\epsilon^{\prime 2}$ when considering the observation of a planetary system. For that we use the expression of $S N B$ given by Eq. 45, and consider the favourable case, in $\mathrm{N}$ band, of a planetary system composed of a hot Jupiter-like planet orbiting a M5 star at 0.5 AU. The planetary flux corresponds to a 'condensed' spectrum extracted from Barman et al. ${ }^{13}$, for which the planet has an intrinsic temperature of $500 \mathrm{~K}$. This planetary system is observed by a typical Bracewell interferometer having a baseline of $20 \mathrm{~m}$, a total transmission of about $1 \%$ at $10 \mu \mathrm{m}$, and an instrumental OPD stability of $2 \mathrm{~nm}$ rms. The left panel of Fig. 5 represents the evolution of $S N B$ as a function of the integration time, for different cross talk levels $\epsilon^{\prime 2}$; while the right panel represents the same $S N B$, but represented as a function of the cross talk level $\epsilon^{\prime 2}$ and normalized by the intrinsic $S N B$ value, i.e. without any flux contamination $\left(\epsilon^{\prime 2}=0 \%\right)$.

In Fig. 5, we clearly see the steady decrease of the 'signal to noise and bias' ratio as a function of $\epsilon^{\prime 2}$, given

the dependence of this ratio on the factor $\sqrt{\left(1-2 \sqrt{\left(1-\epsilon^{\prime 2}\right) \epsilon^{\prime 2}}\right)}$. This was expected since the planetary flux gets less transmitted as the stellar leakage decreases, so that the photon noise of the latter always remains large enough to perturb the planet signal and to decrease $S N B_{\text {planet }}$. 

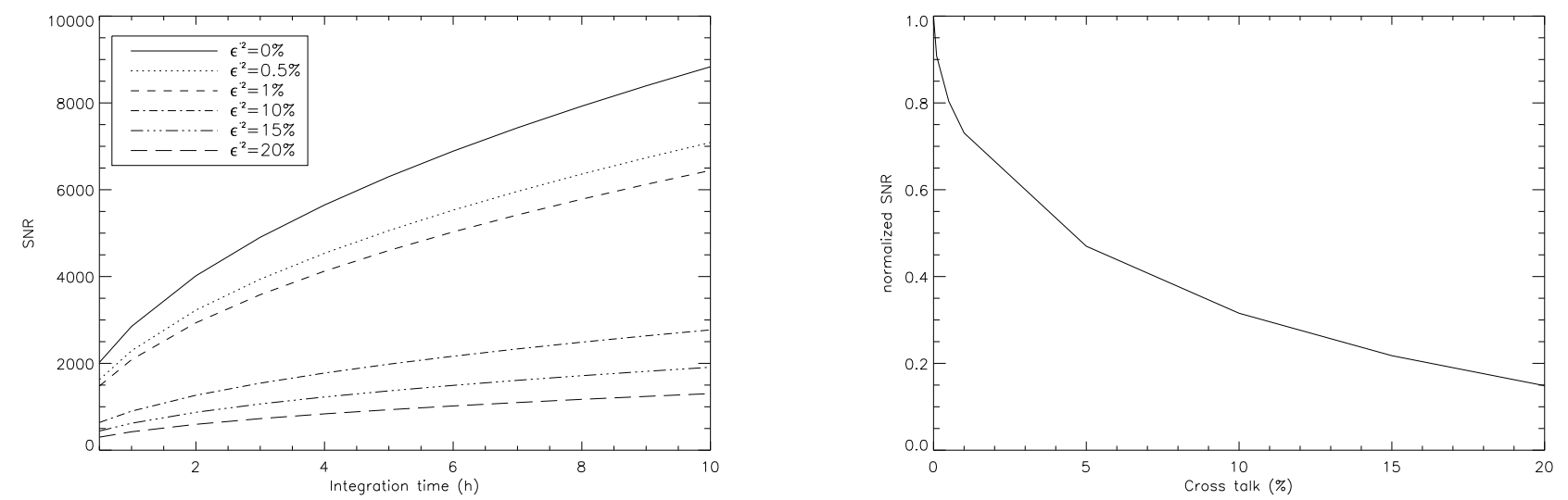

Figure 5. Left panel: Evolution of the 'signal to noise and bias' ratio of the planet null $(S N B)$ as a function of the integration time, for different cross talk levels $\epsilon^{\prime 2}$. Right panel: Evolution of $S N B$ as a function of the cross talk level $\epsilon^{\prime 2} ; S N B$ is normalized to the $S N B$ value without any flux contamination. We assumed the same integration time of one hour.

\subsection{Numerical validation}

In order to validate the theoretical results of the previous section, we used the GENIEsim simulation software ${ }^{15}$ , which was designed to simulate various Bracewell-like interferometer projects such as the GENIE instrument at the VLTI ${ }^{16}$. GENIEsim has the advantage to have been extensively validated by cross-checking with performance estimates done by industrial partners during the GENIE phase A study. It performs end-to-end simulations of nulling interferometers, including the simulation of astronomical sources (star, circumstellar disk, planets, background emission), atmospheric turbulence (piston, longitudinal dispersion, wavefront errors, scintillation), as well as a realistic implementation of closed-loop compensation of phase and intensity perturbations by means of fringe tracking and wavefront correction systems. The output of the simulator basically consists in time series of photo-electrons recorded by the detector at the constructive and destructive outputs of the nulling combiner. The individual signal and noise contributions of the final output GENIEsim are extensively described in Absil et al. ${ }^{15}$ and Defrère et al. ${ }^{16}$.

In the context of this study, we have adapted GENIEsim to handle cross talk by following the mathematical description presented above. This was in fact quite straightforward since only the theoretical expression of the transmission map (Eq. 34) had to be updated. All output signal and noise contributions are then automatically taking cross talk into account. With GENIEsim, we simulated a Bracewell-like interferometer having the same characteristics and specifications as the interferometer described in Section 3.5. We estimated the broadband SNR that would be obtained by such an instrument when observing different types of source, for different values of crosstalk level. These sources are represented by a 5-Zodi exozodiacal disk orbiting a G0V star 10 pc away, a hot Jupiter-like exoplanet (see Section 3.5), and a super-Earth exoplanet, namely Gl 581d, orbiting a M2.5V star 6.26 pc away. Here, we considered significant crosstalk level values up to $5 \%$ in order to clearly emphasize the degradation of the SNR as a function of this parameter. In addition, the crosstalk effect on the remaining instrumental bias is calibrated in our simulations. As a consequence, the broadband SNR (in N band) shown in the following only describes the cross talk effect on the different noise contributions. Fig. 6 shows the broadband SNR for each type of target with respect to the cross talk level $\left(\epsilon^{\prime 2}\right)$. As expected from Eq. 45, with the bias term calibrated, the SNR steadily decreases as a function of the cross talk level. For a significant cross talk level of about $4 \%$, the SNR decreases by about $25 \%$. This decrease is only due to the degradation of the planet/disk signal by a factor $\left(\epsilon-\epsilon^{\prime}\right)^{2}$, as expected from Eq. 40. This effect is partially compensated by the decrease of shot noise due to the improved geometric stellar rejection. Our simulations are thus in good agreement with the theoretical expectations and validate our theoretical description of the parasitic interference problem. However, it is important to remind as well that the significant coherent crosstalk levels considered in our simulations (up to $5 \%$ ), does not correspond to realistic values expected or already estimated in the framework of nulling interferometry testbeds such as PERSEE (see e.g. Jacquinot et al. ${ }^{17}$ ), which is the demonstration bench of 




Figure 6. Simulated broadband SNR of an Bracewell-like interferometer (see Section 3.5 for a description of its characteristics) given with respect to the cross talk level $\left(\epsilon^{\prime 2}\right)$. Three types of source are considered: exozodiacal disk, super-Earth, and hot Jupiter.

the PEGASE and FKSI projects (see Ollivier et al. ${ }^{18}$ and Barry et al. ${ }^{19}$ ), or the Planet Detection Testbed (see Martin et al. ${ }^{9}$ ), which is a demonstration bench for the TPF-I project. For instance, incoherent crosstalk levels of about $10^{-7}$ have been estimated from a ZEMAX model of the PERSEE test bed ${ }^{20}$. Therefore, the phenomenon of parasitic interference does not appear to be problematic and significant in the framework of those current experiments. However, regarding the impact of this phenomenon, a careful attention will anyway have to be paid on the different possible sources of crosstalk, and especially coherent crosstalk, in the design of future nulling interferometry experiments.

\section{CONCLUSION}

In this work we have described the phenomenon of parasitic interference in stellar classical and nulling interferometry. This is the consequence of a coherent crosstalk between beams, occuring through mutual beam contamination or parasitic reflections inside transmitting optics. Through an analytical approach, we have shown that this effect degrades the modulus and the phase of the complex visibility of a classical stellar interferometer, and affects the overall level of the intensity response or transmission map of a nulling interferometer. The two parameters involved in this degradation are the parasitic flux factor (or crosstalk level), and the residual piston between beams before recombination.

In classical stellar interferometry, we have shown that the parasitic phase amplitude is very sensitive to a residual piston between beams. From our feasibility study of hot Jupiter-like planet detection in L band, we concluded that a detection is possible if the parasitic flux reaches at most $5 \%$ of the total incident flux, but only within the residual piston specifications of current fringe trackers. In parallel, we have shown that the closure phase is degraded by this phenomenon as well. The closure phase relation is not zero for an unresolved source and depends on the crosstalk level and the residual piston. This last dependency has to be put in connection with the current piston dependency effects found in the closure phase measurements of the VLTI-AMBER instrument ${ }^{21}$. A study is planned in order to assess the contribution of parasitic interference phenomenon to this piston effect degrading the AMBER data.

In nulling interferometry, parasitic interference (or crosstalk effect) results in a flattening of the transmission map at the destructive output, by a factor $\left(\epsilon-\epsilon^{\prime}\right)^{2}$, in the extreme case of a $\pi$ phase shift between the primary and secondary beams. In our parasitic interference model, we recall that $\epsilon^{2}$ represents the main fraction of each beam, whereas $\epsilon^{\prime 2}$ corresponds to the contaminating fraction of each beam, i.e. the crosstalk level or contamination rate. Because of this flattening of the transmission map, the flux coming from the on-axis stellar source is thus better rejected, while the flux of the off-axis source is less transmitted. As a consequence, the stellar and geometric leakages are decreased by the same factor $\left(\epsilon-\epsilon^{\prime}\right)^{2}$. However, this is counter-balanced by the decrease of the astrophysical signal by the amount, implying a degradation of the final null output SNR, by a factor $\left(\epsilon-\epsilon^{\prime}\right)$. We then validated our analytical study with numerical simulations of the impact of parasitic 
interference on the null output and SNR delivered by a Bracewell-like interferometer. For that, we adapted the GENIEsim simulation software to handle crosstalk. Our results shows that the SNR steadily decreases as a function of the crosstalk level, as expected from our mathematical description. As an exemple, a crosstalk of about $4 \%$ implies a $25 \%$ drop of the SNR on each source. However, as previously mentioned, the parasitic interference phenomenon would appear to be negligible in some current nulling interferometry testbeds such as PERSEE, where very low incoherent crosstalk levels of the order of $10^{-7}$ have been measured.

As a final conclusion, it appears that, up to now, little attention has been paid on the phenomenon of parasitic interference. This issue has been formalized here in a general multi-axial recombination scheme in classical interferometry, with special attention on the differential phase and the closure phase, and in a Bracewell-like scheme in nulling interferometry. For instance, we could see that the detection of astrophysical objects providing weak signatures in the interferometric phase, such as hot Jupiter-like extrasolar planets, requires careful attention to various fine instrumental effects such as parasitic interference. This constitutes an important motivation for optimizing the design of future planet-detecting interferometers like the next ground-based instrument of the VLTI, MATISSE, or the NASA space nulling interferometer project, FKSI. In the context of the MATISSE instrument $^{22}$, Matter et al. ${ }^{5}$ proposed different solutions to prevent crosstalk and thus parasitic interference between beams, especially the separation of the path of each beam by a careful baffling inside the instrument. Solutions that might be implemented into the design of future interferometric instruments.

\section{REFERENCES}

1. H. Fizeau C.R. Acad. Sc. Paris 66, p. 932, 1868.

2. A. A. Michelson, "On the Application of Interference Methods to Astronomical Measurements," ApJ 51, pp. 257-+, June 1920 .

3. A. Labeyrie, "Interference fringes obtained on VEGA with two optical telescopes," ApJ 196, pp. L71-L75, Mar. 1975.

4. R. N. Bracewell, "Detecting nonsolar planets by spinning infrared interferometer," Nature 274, p. 780, Aug. 1978.

5. A. Matter, B. Lopez, S. Lagarde, W. C. Danchi, S. Robbe-Dubois, R. G. Petrov, and R. Navarro, "Parasitic Interference in Long Baseline Optical Interferometry: Requirements for Hot Jupiter-like Planet Detection," ApJ 706, pp. 1299-1308, Dec. 2009.

6. D. Segransan, J. Beuzit, X. Delfosse, T. Forveille, M. Mayor, C. Perrier-Bellet, and F. Allard, "How AMBER will contribute to the search for brown dwarfs and extrasolar giant planets," in Society of Photo-Optical Instrumentation Engineers (SPIE) Conference Series, P. Léna \& A. Quirrenbach, ed., Society of PhotoOptical Instrumentation Engineers (SPIE) Conference Series 4006, pp. 269-276, July 2000.

7. M. Ollivier, Contribution à la recherhce d'exoplanètes - Coronographie interférentielle pour la mission DARWIN. PhD thesis, Université de Paris XI, 1999.

8. B. Chazelas, F. Brachet, P. Bordé, B. Mennesson, M. Ollivier, O. Absil, A. Labèque, C. Valette, and A. Léger, "Instrumental stability requirements for exoplanet detection with a nulling interferometer: variability noise as a central issue," AO 45, pp. 984-992, Feb. 2006.

9. S. R. Martin and A. J. Booth, "Demonstration of exoplanet detection using an infrared telescope array," $A \& A$ 520, p. A96, Sept. 2010.

10. O. P. Lay, "Systematic Errors in Nulling Interferometers," AO 43, pp. 6100-6123, Nov. 2004.

11. N. M. Elias, II, M. Harwit, D. Leisawitz, and S. A. Rinehart, "The Mathematics of Double-Fourier Interferometers," ApJ 657, pp. 1178-1200, Mar. 2007.

12. M. Vannier, Interférométrie et astrométrie différentielle chromatiques et observation de planètes extrasolaires géantes chaudes avec le VLTI et le NGST. PhD thesis, Laboratoire Universitaire d'Astrophysique de Nice/Université de Nice-Sophia Antipolis, mai 2003.

13. T. S. Barman, P. H. Hauschildt, and F. Allard, "Irradiated Planets," ApJ 556, pp. 885-895, Aug. 2001.

14. O. Absil, Astophysical studies of extrasolar planetary systems using infrared interferometric techniques. $\mathrm{PhD}$ thesis, Liège University, Liège, Belgium, 2006. 
15. O. Absil, R. den Hartog, P. Gondoin, P. Fabry, R. Wilhelm, P. Gitton, and F. Puech, "Performance study of ground-based infrared Bracewell interferometers. Application to the detection of exozodiacal dust disks with GENIE," A\&̈A 448, pp. 787-800, Mar. 2006.

16. D. Defrère, O. Absil, V. Coudé du Foresto, W. C. Danchi, and R. den Hartog, "Nulling interferometry: performance comparison between space and ground-based sites for exozodiacal disc detection," $A \mathscr{E} A 4 \mathbf{4 9 0}$, pp. $435-445$, Oct. 2008.

17. S. Jacquinod, F. Cassaing, J.-M. Le Duigou, M. Barillot, M. Ollivier, K. Houairi, F. Lemarquis, and J.P. Amans, "PERSEE: description of a new concept for nulling interferometry recombination and OPD measurement," in Society of Photo-Optical Instrumentation Engineers (SPIE) Conference Series, Society of Photo-Optical Instrumentation Engineers (SPIE) Conference Series 7013, July 2008.

18. M. Ollivier, O. Absil, F. Allard, J.-P. Berger, P. Bordé, F. Cassaing, B. Chazelas, A. Chelli, O. Chesneau, V. Coudé du Foresto, D. Defrère, P. Duchon, P. Gabor, J. Gay, E. Herwats, S. Jacquinod, P. Kern, P. Kervella, J.-M. Le Duigou, A. Léger, B. Lopez, F. Malbet, D. Mourard, D. Pelat, G. Perrin, Y. Rabbia, D. Rouan, J.-M. Reiss, G. Rousset, F. Selsis, P. Stee, and J. Surdej, "PEGASE, an infrared interferometer to study stellar environments and low mass companions around nearby stars," Experimental Astronomy 23, pp. 403-434, Mar. 2009.

19. R. K. Barry, W. C. Danchi, B. Lopez, S. A. Rinehart, O. Absil, J.-C. Augereau, H. Beust, X. Bonfils, P. Bordé, D. Defrère, P. Kern, A. Léger, J. Monin, D. Mourard, M. Ollivier, R. Petrov, F. Vakili, and FKSO Consortium, "The Fourier-Kelvin Stellar Interferometer: Exploring Exoplanetary Systems with an Infrared Probe-class Mission," in Pathways Towards Habitable Planets, V. Coudé Du Foresto, D. M. Gelino, \& I. Ribas, ed., Astronomical Society of the Pacific Conference Series 430, pp. 403-+, Oct. 2010.

20. S. Jacquinot, Interférométrie annulante pour l'exoplanétologie - Etude et développement du recombinateur du banc Persée. PhD thesis, Université Paris 11, mars 2010.

21. M. Vannier, et al., "High-precision closure phase for optical interferometry," in Society of Photo-Optical Instrumentation Engineers (SPIE) Conference Series, Society of Photo-Optical Instrumentation Engineers (SPIE) Conference Series, 2012.

22. B. Lopez, et al., "MATISSE: perspective of imaging in the mid-infrared at the VLTI," in Society of PhotoOptical Instrumentation Engineers (SPIE) Conference Series, Society of Photo-Optical Instrumentation Engineers (SPIE) Conference Series 7013, July 2008. 